\title{
A proposito di...diete iperproteiche: proviamo a discuterne
}

\author{
Luisa Paschino
}

Casa di Cura “Villa Igea”, Servizio di Dietetica e Nutrizione Clinica, Acqui Terme (AL)

\begin{abstract}
About... High-PRotein Diets: LeT'S discuss ABOUT IT
Abstract. Considering the continuous increase of overweight and obesity cases all over the world, the general population shows greater interest in the use of High-Protein diets to contrast this phenomenon.

There are still several doubts about the long-term safety of High-Protein diets, especially with regard to the potential development and progression of Chronic Kidney Disease (CKD).

High-Protein diets have been correlated to kidney hypertrophy, glomerular hyperfiltration, CKD acceleration, increased proteinuria, greater risk of nephrolithiasis and various metabolic changes.

While it is now generally accepted that these conditions may determine progressive kidney failure in individuals already suffering from CKD, the consequences of a high protein intake on healthy subjects is less clear.

A global understanding of the risks related to High-Protein diets is still limited due the lack of both rigorous long-term studies on humans and a universally recognised definition of High-Protein diet.

For this reasons, High-Protein diets are not recommended for people with GFR $<60 \mathrm{~mL} / \mathrm{min}$, and healthy subjects should first undergo a screening test to measure serum creatinine and microalbuminuria.

Thus, considering that most of the studies and meta-analysis published until now show that High-Protein diets are neither salutary nor detrimental in terms of long-term weight loss, it seems premature to recommend such diets for the treatment of overweight and obesity conditions.
\end{abstract}

Key words: High-Protein diets, CKD, Weight loss, Popular diets, Obesity

Conflict of interest: None.

Financial support: None.

Accettato: 23 Giugno 2014

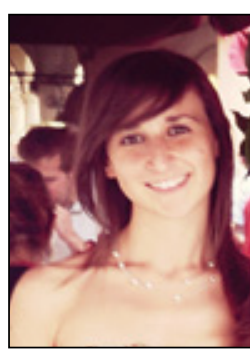

Luisa Paschino

\section{Introduzione}

Nella letteratura popolare la prima dieta iperproteica elaborata per la perdita di peso è stata descritta da William Banting nel 1860: "Il più grande fascino e conforto di questo sistema è che gli effetti sono evidenti nell'arco di una settimana e creano uno stimolo per continuare per altre settimane" (1).

La prima evidenza sulla perdita di peso basata su diete iperproteiche (Low-Carbohydrate, High-Protein diets) risale invece al 1994. Da questo momento in poi, esplode l'attenzione della popolazione generale e dei professionisti sanitari nei confronti di questi regimi nutrizionali, spinta in particolare dalla pubblicazione di libri sulla dieta Atkins $(2,3)$.

Specialmente negli ultimi decenni, ed in risposta alla crescente incidenza di obesità, c'è stato un aumento dell'interesse pubblico all'uso di metodi alternativi per la perdita di peso che sono in contrasto con le raccomandazioni delle Linee Guida Nutrizionali (4).

Solo negli Stati Uniti le persone spendono circa 33 miliardi di dollari all'anno in programmi per la perdita di peso, prodotti e pillole. Libri sull'argomento appaiono continuamente e diventano in poco tempo dei best-seller: la Dieta del Minestrone, la dieta del Pompelmo, la New Beverly Hills diet, la Dieta Zona, la Atkins e la dieta Dukan rappresentano solo alcuni esempi delle diete appartenenti al cosiddetto mondo delle "popular diets" o "diet industry" fra cui il consumatore può oggi scegliere (5). Tali diete attirano l'attenzione poiché, non solo promettono il calo ponderale, ma lo assicurano anche in tempi rapidi. In effetti, alcune evidenze mostrano migliori risultati a breve termine ma ci sono preoccupazioni sull'utilità e sulla sicurezza delle diete iperproteiche soprattutto nei soggetti affetti da diabete mellito di tipo II, ipertensione, dislipidemia, cardiopatia ischemica e malattia renale cronica (CHD) $(1,6)$. Vi sono inoltre pareri 
discordanti nel ritenere l'eccessivo apporto di proteine un potenziale fattore di rischio per lo sviluppo della patologia renale negli individui sani (7).

\section{Diete iperproteiche: classificazione}

Esistono diversi modi di menzionare in letteratura diete a basso apporto di carboidrati e/o ad elevato apporto di proteine e/o chetogeniche:

- VLCD (Very Low Calorie Diet): dieta con apporto calorico inferiore alle $800 \mathrm{Kcal}$;

- Low-Carbohydrate Diet: dieta con apporto calorico estremamente ristretto, con riduzione del consumo di carboidrati a $20-60 \mathrm{~g} / \mathrm{die}$;

- VLCKD (Very Low-Carbohydrate Ketogenic Diet): dieta con apporto calorico estremamente ristretto, con riduzione del consumo di carboidrati a 20-80 g/die;

- Diete chetogeniche: utilizzate sin dagli anni '20 nel trattamento dell'epilessia infantile farmaco-resistente, con un rapporto chetogenico 4:1 (4 g di grassi per ogni g di carboidrati);

- High-Protein Diet: dieta con apporto proteico in grado di coprire dal $20 \%$ al $45 \%$ delle calorie totali (90-200 g/die, a seconda degli studi);

- NEC (Nutrizione Enterale Chetogena): somministrazione di una soluzione di elettroliti e amminoacidi (35-40 g) tramite una sonda naso-gastrica che non dovrebbe essere mai rimossa durante i 10 giorni del ciclo di trattamento. Sembrerebbe che sia il paziente a decidere il numero dei cicli (8).

\section{Meccanismo d'azione delle diete iperproteiche}

Le diete iperproteiche possono influenzare il bilancio energetico aumentando il senso di sazietà e aumentando il BEE (Basal Energy Expenditure): questi fattori possono spiegare gli effetti delle proteine sulla perdita di peso e sulla perdita di massa grassa.

Molti studi hanno mostrato che i soggetti riferiscono maggior senso di sazietà dopo aver consumato un pasto ad elevato contenuto proteico rispetto ai classici pasti con elevato apporto di carboidrati; in alcuni casi vi è un aumento della spesa energetica postprandiale o riduzione dell'intake al pasto successivo. Un elevato apporto di proteine con la dieta potrebbe aumentare il BEE attraverso l'aumento della termogenesi indotta dalla dieta (DIT): essa, infatti, è maggiore del $17 \%$ nei pasti ricchi di proteine rispetto a quelli ricchi di carboidrati e di grassi. Inoltre, le proteine animali aumentano maggiormente la spesa energetica rispetto a quelle di origine vegetale.

La differente composizione in amminoacidi degli alimenti può avere effetti diversi sulle concentrazioni di ormoni associati alla regolazione dell'appetito come colecistochinina (CCK), GLP-1 e peptide tirosina-tirosina. Allo stesso modo sembrerebbe che specifici amminoacidi nella dieta siano coinvolti nella produzione di neurotrasmettitori, anch'essi implicati nella regolazione dell'appetito. Gli amminoacidi ramificati (BCAA), ed in particolare la leucina, rivestono un ruolo chiave nel metabolismo muscolare e agiscono come regolatori e come substrato per la sintesi delle proteine muscolari (9).

Oltre agli aspetti sinora citati, si è ormai consolidato negli anni che il razionale principale alla base della forte restrizione dei carboidrati nella dieta sia dovuto alla risultante chetosi, la quale diminuisce i livelli basali di insulina in modo tale da promuovere la lipolisi, diminuire la lipogenesi e incrementare dunque la perdita di massa grassa.

Dopo pochi giorni di digiuno o di drastica riduzione dell'apporto di carboidrati ( $<50 \mathrm{~g} /$ die), le riserve di glucosio diventano insufficienti sia per la normale ossidazione dei grassi (attraverso la fornitura di ossalacetato al ciclo di Krebs) sia per la fornitura di glucosio al sistema nervoso centrale (SNC). Il SNC è così "costretto" a trovare fonti energetiche alternative e, come avevano dimostrato gli esperimenti di Cahill e colleghi nel 1967, tale energia alternativa deriva dalla produzione di acetil-CoA in eccesso. Questa condizione osservata nel digiuno prolungato, nel diabete di tipo 1 e nelle diete a basso apporto di carboidrati o iperproteiche, porta alla produzione di corpi chetonici (acetone, acetoacetato e $\beta$ - idrossibutirrato), un processo denominato chetogenesi. I chetoni circolanti, inoltre, sopprimono l'appetito dell'individuo, imitando un vero e proprio stato di anoressia (10-12).

\section{Diete iperproteiche: calo ponderale e salute}

La maggior parte degli studi sino ad ora pubblicati dimostra un miglior calo ponderale a breve termine nei gruppi a dieta iperproteica rispetto ai gruppi di controllo, tuttavia dopo un solo anno di follow-up la differenza non rimane statisticamente significativa. In tali studi solitamente viene modificata la percentuale di macronutrienti, mentre l'apporto e la restrizione di energia sono i medesimi in tutti i gruppi in analisi $(2,9)$.

In uno studio condotto da Skov et al sono state messe a confronto due diete popolari (Atkins e Zona) con una dieta convenzionale a basso apporto di grassi ed elevato apporto di carboidrati. Dopo 6 mesi gli individui appartenenti al gruppo della dieta Atkins e Zona avevano ottenuto maggior calo ponderale e riduzione dei trigliceridi rispetto a coloro che seguivano una dieta ad elevato apporto di carboidrati.

Tale differenza scompariva dopo un anno, anche se i soggetti che avevano raggiunto un calo ponderale del $10 \%$ erano maggiormente rappresentati nei gruppi a dieta iperproteica (9). Il più grande studio Americano sulle diete iperproteiche è stato condotto in USA in quattro centri; i partecipanti sono stati suddivisi random fra tre possibili diete (a medio apporto di proteine, $15 \%$, ad elevato apporto di proteine, $25 \%$, ad elevato apporto di proteine, $25 \%$, e di lipidi, $40 \%$, con una riduzione calorica di $750 \mathrm{Kcal}$ rispetto al dispendio energetico) da seguire per 2 anni. A 6 mesi la perdita di peso era di circa il $6 \%-7 \%$ in tutti i gruppi, a 12 mesi i soggetti riprendevano peso e a 2 anni non c'era differenza statisticamente significativa tra le diverse diete (2). Nello specifico, è stato mostrato che la compliance a regimi dietetici molto severi diminuisce a breve-medio termine rispetto alle diete convenzionali. In alcune ricerche scientifiche in cui l'assunzione proteica da parte dei partecipanti è stata controllata con la misurazione dell'azoturia delle $24 \mathrm{~h}$ e il calcolo della Protein Catabolic Rate (PCR), si è evidenziato come l'apporto proteico si riduca nei gruppi tenuti a dieta iperproteica sino a divenire del tutto sovrapponibile a quello dei gruppi di controllo, tenuti invece a dieta con 
normale apporto di carboidrati e proteine $(2,13)$. Ad Agosto 2012, sull'American Journal of Clinical Nutrition, è stata pubblicata una meta-analisi di studi randomizzati controllati la quale ha confermato che, comparata ad una dieta ad apporto proteico standard, una dieta iperproteica isocalorica dà modesti benefici in termine di riduzione di peso, massa grassa e trigliceridi, con una minore riduzione di massa magra e di BEE. Nel lungo termine, invece, gli effetti di queste diete sul calo ponderale e sul rischio cardiovascolare rimangono quasi del tutto sconosciuti (14).

È stato riportato un aumento del rischio di tutte le cause di morte e di diabete di tipo 2 con diete LCHP (Low-Carbohydrate, High-Protein) se seguite a lungo termine anche nell'individuo sano, con assunzione proteica pari al 20\%-23\% dell'energia totale. Gli effetti di apporti proteici superiori restano da indagare (15).

Infine, Schwinghckl e Hoffman, in una meta-analisi pubblicata ad Agosto 2013 dove sono state messe a confronto diete HP (High-Protein, $\geq 25 \%$ dell'energia totale) con diete LP (Low-Protein, $<20 \%$ dell'energia totale) seguite per un periodo di almeno 12 mesi, hanno concluso che le diete iperproteiche non modificano i markers di obesità, patologia cardiovascolare e controllo glicemico. Tenendo conto dei potenziali rischi correlati ad un elevato apporto di proteine, appare perciò prematuro raccomandare queste diete per il trattamento di sovrappeso e obesità (16).

\section{Potenziali effetti sul rene nell'individuo non affetto da CKD}

Articoli risalenti al 1917 hanno valutato l'influenza di una dieta ad elevato contenuto di proteine sul rene, evidenziando un effetto dannoso soprattutto in caso di malattia renale. In questi studi si era già visto come fosse diverso l'impatto sul rene a seconda che le proteine fossero di origine animale o vegetale (17).

Le diete iperproteiche sono state associate a ipertrofia renale, iperfiltrazione glomerulare, accelerazione della CKD, aumento della proteinuria, maggior rischio di nefrolitiasi e varie alterazioni metaboliche. In particolare, l'intake di proteine aumenta sia negli animali che nell'uomo il flusso plasmatico renale e l'eGFR sino al $100 \%$ rispetto al livello basale e sembra incidere anche sul volume e sul peso dei reni. Le proteine animali hanno un effetto maggiore sull'emodinamica del rene, seguite dalle lattoproteine e, infine, da quelle vegetali.

Nonostante i cambiamenti emodinamici servano al rene proprio per eliminare il carico proteico, questo potrebbe essere deleterio nel lungo termine.

Brenner et al hanno condotto studi sperimentali dimostrando che l'aumento della pressione intraglomerulare nel tempo porta a sclerosi glomerulare, particolarmente nei reni di animali già malati o danneggiati. Non è ancora chiaro invece l'effetto dell'assunzione di diete iperproteiche sul rene sano nel lungo termine (18).

The Nurse's Health Study (19) ha confermato che nelle donne con funzione renale normale seguite per un periodo di 11 anni, il consumo di proteine nella dieta non esercitava nessun effetto sulla funzione renale. Viceversa, nelle donne affette da CKD lieve (stadio 1-2), una dieta iperproteica, soprattutto di origine animale (escludendo i derivati del latte), poteva accelerare l'evoluzione della nefropatia.

Nei modelli sperimentali di uremia e nei pazienti affetti da CKD di stadio 4-5, l'accumulo di tossine derivanti dal catabolismo di un carico di proteine peggiora la sindrome uremica, lo stress ossidativo, la disfunzione endoteliale, la resistenza insulinica, ecc (20).

I reni di ratti mantenuti a dieta iperproteica $(30 \%$ dell'energia totale) presentano un'infiltrazione interstiziale di linfociti $\mathrm{T}$ e macrofagi e presenza di tessuto fibrotico. La terapia immunosoppressiva attenua l'ipertensione arteriosa e il danno renale e ciò indica che l'infiltrazione di queste cellule gioca un ruolo patologico nei ratti alimentati con dieta iperproteica: è stato ipotizzato che queste cellule abbiano effetti deleteri sul rene favorendo il rilascio di radicali liberi, citochine e altri fattori vasoattivi (21).

Lo studio OMNI- Heart (Optimal Macro-Nutrient Intake) valuta l'effetto di una parziale sostituzione dei carboidrati con le proteine sulla funzionalità renale di individui sani, utilizzando nuovi markers. Quando i soggetti in esame sono stati tenuti a dieta iperproteica si evidenziava un aumento dell'eGFR e una diminuzione dei livelli di Cistatina C e B2-microglobulina, mentre si registrava un aumento della creatininemia. Limite di tale studio, oltre che la breve durata, è che una buona quota delle proteine in eccesso è stata somministrata sotto forma di proteine di origine vegetale e, quindi, i risultati non corrispondono direttamente a quelli che si otterrebbero con le cosiddette diete popolari (22).

Nelle diete iperproteiche, inoltre, aumenta il rischio di microalbuminuria soprattutto in soggetti con diabete e ipertensione arteriosa (18). Nonostante la maggior parte degli studi presenti in letteratura non sono in accordo nel considerare le diete iperproteiche come potenziale fattore di rischio di nefrolitiasi, le evidenze mostrano che un elevato consumo di proteine porta ad iperuricosuria, ipercalciuria, ipocitraturia e diminuzione del $\mathrm{pH}$ urinario. Le High-Protein diets danno inoltre un carico acido che si può valutare attraverso la diminuzione del $\mathrm{pH}$ urinario, l'aumento dell'escrezione di ammonio, l'ipocitraturia e l'ipercalciuria. Il carico acido sembra però ben tollerato dagli individui sani in quanto non si denota la presenza di acidosi metabolica (23).

Borghi et al hanno dimostrato come le diete a basso contenuto proteico e sodico, con normali livelli di calcio, riducano invece la comparsa di calcoli in soggetti con calcolosi di ossalato di calcio ricorrente e ipercalciuria (24). La dieta iperproteica sembrerebbe favorire inoltre un bilancio negativo del calcio, in quanto la frazione di calcio assorbita a livello intestinale rimane pressoché invariata, mentre ne aumenta l'escrezione urinaria. In aggiunta, nei soggetti con aumentato apporto di proteine sembrano diminuire i livelli di osteocalcina (23). L'osso, però, è un tessuto complesso che cambia lentamente $\mathrm{e}$, come tale, è difficile quantificare gli effetti dei nutrienti su di esso (25).

Gli studi condotti su soggetti obesi mostrano invece miglioramento della funzionalità renale (riduzione dell'iperfiltrazione, diminuzione della microalbuminuria e della resistenza insulinica) indipendentemente dal tipo di dieta ipocalorica seguita. 
Ciò sottolinea come l'obesità sia un fattore di rischio indipendente di CKD ed evidenzia l'importanza del calo ponderale nel rallentare la progressione della patologia renale nelle primissime fasi di malattia $(26,27)$.

Una comprensione globale del rischio associato a tali diete è ancora oggi limitata dalla mancanza di una definizione universalmente accettata di High-Protein diet e dall'assenza di rigorosi studi a lungo termine sull'uomo. Ci si affida perciò a studi condotti sugli animali oppure a studi a breve termine, in cui la valutazione diviene molto difficile poiché l'impatto renale di un elevato intake proteico sarà probabilmente diverso per periodi limitati piuttosto che per periodi prolungati di consumo.

È stato ormai accettato che l'iperfiltrazione glomerulare possa accelerare la progressione del danno renale nelle persone che già soffrono di patologia renale. Così come citato in precedenza, è invece meno chiaro l'impatto di un elevato apporto di proteine nei soggetti sani, soprattutto per quanto riguarda l'età degli individui: essa è infatti associata ad un declino fisiologico della funzione renale in termine di diminuzione del GFR e diminuzione dell'abilità renale nell'escrezione di un carico acido. Poiché la CKD è spesso una patologia silente, tutti, prima di intraprendere tali diete, dovrebbero essere sottoposti a uno screening con misurazione della creatininemia e utilizzo di dipstick per la valutazione della presenza di proteinuria. Particolare attenzione dev'essere riservata agli individui affetti da sovrappeso, diabete e ipertensione poiché ne sono particolarmente vulnerabili $(7,18)$.

\section{Conclusioni}

La prevalenza di obesità e sovrappeso in costante aumento ha incrementato l'interesse verso le cosiddette Popular Diets, generalmente ipocaloriche e iperproteiche. Solo negli Stati Uniti le persone spendono circa 33 miliardi di dollari all'anno in programmi per la perdita di peso, prodotti e pillole.

Nonostante il calo ponderale sia maggiore nelle diete iperproteiche, ad un anno di follow-up, la perdita di peso risulta la medesima se confrontata con diete convenzionali basate sulle Linee Guida Nutrizionali. L'effetto a lungo termine dell'assunzione di diete iperproteiche sul rene non è ancora chiaro. Una comprensione globale del rischio associato a tali diete è limitata dalla mancanza di una definizione universalmente accettata di High-Protein diet e dall'assenza di rigorosi studi a lungo termine sull'uomo. Dall'analisi di queste diete si evince come sia diverso l'apporto proteico, passando dal $25 \%$ dell'energia totale sino ad arrivare al $50 \%$.

Ad oggi, non esistono chiare controindicazioni alle diete iperproteiche nei soggetti sani ma ciò dovrebbe essere approfondito. Viceversa, i potenziali rischi nelle persone con CKD sono fondati e includono la progressione accelerata della preesistente CKD, aumento della proteinuria, disordini idro-elettrolitici, maggior tendenza alla formazione di calcoli e aggravamento dei sintomi uremici; tali diete vanno quindi evitate in questi individui.

Poiché non è stato ancora determinato in quali casi sia meglio non raccomandare le diete iperproteiche, è ragionevole evitarle nei soggetti con GFR $<60 \mathrm{~mL} / \mathrm{min}$ o in presenza di sindrome nefrosica.
Poiché la CKD è spesso una patologia silenziosa, per i soggetti in apparente buona salute è bene effettuare uno screening con misurazione della creatininemia e utilizzo di dipstick per la valutazione della presenza di proteinuria prima di affidarsi ad una Popular Diet, così come suggerito dalla National Kidney Foundation. Ancor meglio dell'utilizzo di dipstick sarebbe l'effettiva misurazione della microalbuminuria.

\section{Riassunto}

Con l'aumento costante della prevalenza di sovrappeso e obesità in tutto il mondo, è sempre maggiore l'interesse e l'utilizzo di diete iperproteiche da parte della popolazione generale per contrastare tale fenomeno. Tuttavia, vi sono ancora oggi forti dubbi sulla sicurezza a lungo termine delle High-Protein diets, soprattutto per quanto riguarda il possibile sviluppo e la progressione della patologia renale.

Le diete iperproteiche sono state associate a ipertrofia renale, iperfiltrazione glomerulare, accelerazione della CKD, aumento della proteinuria, maggior rischio di nefrolitiasi e varie alterazioni metaboliche. Mentre è ormai accettato che tali condizioni possono causare un danno renale progressivo nelle persone che già soffrono di patologia renale, meno chiaro è invece l'impatto di un elevato apporto di proteine nei soggetti sani. Una comprensione globale del rischio ad esse correlato è ancora oggi limitata dall'assenza di rigorosi studi a lungo termine sull'uomo e dalla mancanza di una definizione universalmente accettata di High-Protein diet. A tal proposito è bene non indicare diete iperproteiche nei soggetti con GFR $<60 \mathrm{~mL} / \mathrm{min}$, mentre, per quanto riguarda gli individui sani, essi dovrebbero essere sottoposti ad uno screening con misurazione della cratininemia e della microalbuminuria prima di affidarsi a queste diete.

Tenendo conto che la maggior parte degli studi e delle meta-analisi sino ad ora pubblicate mostrano che le diete iperproteiche non sono né benefiche né dannose in termine di calo ponderale a lungo termine, appare comunque prematuro raccomandare tali diete per il trattamento di sovrappeso e obesità.

Parole chiave: Diete iperproteiche, CKD, Calo ponderale, Popular diets, Obesità

\section{Consenso informato: N/A}

Dichiarazione di conflitto di interessi: L'Autore dichiara di non avere conflitto di interessi.

Contributi economici agli Autori: L'Autore dichiara di non aver ricevuto sponsorizzazioni economiche per la preparazione dell'articolo.

Indirizzo dell'Autore:

Luisa Paschino

Via Terracini 6

15067 Novi Ligure (AL)

luisa.paschino@hotmail.it 


\section{Bibliografia}

1. Bravata DM, Sanders L, Huang J, et al. Efficacy and Safety of Low-Carboydrate Diets. JAMA. 2003; 289(14): 1837-49.

2. Freedman MR, King J, Kennedy E. Popular diets: a scientific review. Ob Res. 2001; 9: 1-40.

3. Clifton P. Effects of a high protein diet on body weight and comorbidities associated with obesity. Br J Nutr. 2012; 108: 122-9.

4. Westman EC, Feinman RD, Mavropoulos JC, et al. Lowcarbohydrate nutrition and metabolism. Am J Clin Nutr. 2007; 86: $276-84$

5. Stein K. High-protein, low-carbohydrate diets: Do they work? J Am Diet Assoc. July 2000; 100(7): 11.

6. Malik VS, Hu FB. Popular weight-loss diets: from evidence to practice. Nat Rev Cardiol. 2007; 4(1): 34-41.

7. Wagner EA, Falciglia GA, Amlal H, Levin L, Soleimani M. Short-term exposure to a high-protein diet differentially affects glomerular filtration rate but not acid-base balance in older compared to younger adults. J Am Diet Assoc. 2007; 107(8): 1404-8.

8. Donini LM, Sandri G, Fusco MA, Muscaritoli M. Position Statement dell Fe.S.I.N. sulla cosiddetta Nutrizione Enterale Chetogena (NEC) come terapia dell'obesità. Società Italiana dell'Obesità. Dicembre 2012.

9. Te Morenga L, Mann J. The role of high-protein diets in body weight management and health. Br J Nutr. 2012; 108: 130-8.

10. Poli A, Rubini A, Volek JS, Grimaldi KA. Beyond weight loss: a review of the therapeutic uses of very-low-carbohydrate (ketogenic) diets. Eur J Clin Nutr. 2013; 67: 789-96.

11. Johnstone AM, Horgan GW, Murison SD, Bremner DM, Lobley GE. Effects of a high-protein ketogenic diet on hunger, appetite, and weight loss in obese men feeding and libitum. Am J Clin Nutr. 2008; 87: 44-55.

12. Astrup A, Larsen TM, Harper A. Atkins and other lowcarbohydrate diets: hoax or an effective tool for weight loss? Lancet. 2004; 364: 897-9.

13. Dansinger ML, Gleason JA, Griffith JL, Selker HP, Schaefer EJ. Comparison of the Atkins, Ornish, Weight Watchers and Zone Diets for weight loss and heart disease risk reduction. JAMA. 2005; 293(1): 43-53.

14. Wycherley TP, Moran LJ, Clifton PM, Noakes M, Brinkworth GD. Effects of energy-restricted high-protein, low-fat compared with standard-protein, low-fat diets: a meta-analysis of randomized controlled trials. Am J Clin Nutr. 2012; 96: 1281-98.
15. Pedersen AN, Kondrup J, Borsheim E. Health effects of protein intake in healthy adults: a systematic literature review. J Food Nutr Res. 2013; 57: 1-29.

16. Schwingshackl L, Hoffmann G. Long-term effects of lowfat diets either low or high in protein on cardiovascular and metabolic risk factors: a systematic review and meta-analysis. J Nutr. 2013; 12: 48.

17. Lewis DS. On the influence of a diet with high protein content on the kidney. Can Med Assoc J Arch Int Med 1917; 19: 682-3.

18. Friedman AN. High-Protein Diets: potential effects on kidney in renal health and disease. Am J Kidney Dis. 2004; 44(6): 950-62.

19. Knight EL, Stamfer MJ, Hankinson SE, Spigelman D, Curhan GC. The impact of protein intake on renal function decline in women with normal renal function or mild renal insufficiency. Ann Intern Med. 2003; 138(6): 460-7.

20. Chauveau P, Comble C, Aparicio M, Fouque D. Il consumo di proteine nell'insufficienza renale cronica. G Ital Nefrol 2013; 30(5). pii:gin/30.5.11.

21. De Miguel C, Lund H, Mattson DL. High Dietary Protein exacerbates hypertension and renal damage in Dahl SS rats by increasing infiltrating immune cells in the kidney. Hypertension. 2011; 57(2): 269-74.

22. Juraschek SP, Appel LJ, Anderson CA, Miller ER. Effect of a High-Protein Diet on Kidney Function in healthy adults: results from the OmniHeart Trial. Am J Kidney Dis. 2013; 61(4): 547-54.

23. Reddy TS, Wang CY, Sakhaee K, Brinkley L, Pak CYC. Effect of low-carbohydrate high-protein diets on acid-base balance, stone-forming propensity, and calcium metabolism. Am J Kidney DiS. 2002; 40(2): 265-74.

24. Borghi L, Schianchi T, Meschi T, et al. Comparison of two diets for prevention of recurrent stones in idiopathic hypercalciuria. N Engl J Med. 2002; 346(2): 77-84.

25. Kestetter JE, Brien K, Insogna K. Dietary protein, calcium metabolism, and skeletal homeostasis revisited. Am J Clin Nutr. 2003; 78(suppl): 584-92.

26. Brinkworth GD, Buckley JD, Noakes M, Clifton PM. Renal function following long-term weight loss in individuals with abdominal obesity on a Very-Low-Carbohydrate Diet vs HighCarbohydrate Diet. J Am Diet Assoc. 2010; 110(4): 633-38.

27. Tirosh A, Golan R, Harman-Boehm I, et al. Renal function following three distinct weight loss dietary strategies during 2 years of a randomized controlled trial. Diabetes Care. 2013; 32: 2225-32. 